\title{
Unilateral Teleoperation Design for a Robotic Endoscopic Pituitary Surgery System
}

\author{
M. İ. C. Dede ${ }^{1}$, O. W. Maaroof ${ }^{1}$, G. Ateş ${ }^{1}$, M. Berker ${ }^{2}$, İ. Işıkay ${ }^{2}$ and Ş. \\ Hanalioğlu ${ }^{2}$ \\ ${ }^{1}$ Izmır Institute of Technology, Izmir, Turkey, e-mail:candede@iyte.edu.tr, \\ omarmaaroof@iyte.edu.tr,gizemates@iyte.edu.tr \\ ${ }^{2}$ Hacettepe University,Ankara,Turkey,e-mail:mberker@hacettepe.edu.tr, \\ ilkayisikay@gmail.com,sahinhanalioglu@gmail.com
}

\begin{abstract}
The aim of this study is to develop a teleoperation system which will be used to support the endoscopic pituitary surgery procedures. The proposed system aims to enable the surgeon to operate with three different operation tools (one of them is the endoscope) simultaneously. By this way, it is expected that the productivity of the surgical operation will be improved and the duration of the operation will be shortened. In the proposed system, a main control unit that can be attached to any of the surgical tools that are used in the operation (other than the endoscope) will be developed to capture the motion of the surgeon's hand motion as demanded by the surgeon, to process the captured motion and to send it to the robot that handles the endoscope. In this way, the endoscope will be directed simultaneously by the surgeon throughout the operation while he/she is using the other surgical tools with his/her two hands. In this paper, the study to determine the type and processing of information that is sent from the surgeon's side to the endoscope robot is presented.
\end{abstract}

Keywords: endoscopic pituitary surgery, robotic surgery, pituitary surgery, minimally invasive surgery, teleoperation.

\section{Introduction}

In last 20 years, many groups have been working on controlling motion of medical imaging systems such as laparoscope, endoscope by robotic systems. In order to reduce the amount of people in surgeries and reduce the duration, controlling the robotic system by the surgeon is preferred. One of the most important points is how the control demands will be sent to the robot. Since the surgeon sends the control demands to the robot, these demands should be acquired in an intuitive way so that the surgeon's concentration will not be deviated from the primary focus, the surgery itself. Being a technological device, these robotic systems bring some technical problems alongside similar to other technical devices that are already in use in all operating rooms. Among these, the most significant one is the 
safety. For safety, some redundant safety mechanisms are utilized in different levels. Another constraint is the need of technical training for the surgeon to use these systems. Additionally, even if robots have reached higher precision levels during operation, they are still slower than the human. Furthermore, robots should occupy as little place as possible in the operation room and whenever it is required they should move or restrain the motion of the optical system. All these topics should be considered in designing a surgical robot independent from the operation type.

Robots are generally categorized into two as the active or the passive robots. Active surgical robot systems are capable of recognizing the changes in the environment and organize their duties accordingly. As an example, ROBODOC [1] is an active robot which is used in femur operations. In passive surgical robot systems (also called the master-slave robot systems), the surgeon provided the motion inputs to the slave system via the master system. The control of the slave system is achieved by using these inputs in its control algorithm during surgery. In addition to these, in some semi-active systems such as NeuroMate [2], the surgeon provides the directions. In another type of robotic surgery, the robot and the surgeon handle and direct the surgical tool together. Mako Surgical Corp. product [3] that is used in arthroscopic knee surgeries is a good example of these type of robots.

One of the important advantages of passive systems is the capability of scaling the motion demands which are received from the master system to increasing the sensitivity of the slave system. The tremor problem in surgeon's hand while holding the endoscope for long hours can be resolved by using the master-slave systems and filtering out the tremors within the master systems. Another advantage is that slave system can have six or more degrees-of-freedom (DoF) and the surgeon can enter inputs not only by his/her hands but also fingers and elbow for the slave system. Therefore, flexibility of the system increases [4].

In the literature, there are neurosurgical systems in which these passive systems are used. One of them is developed by Morita [5] and his group for microscopic neurosurgery. Although this system provides the necessary precision levels, it can be used only by surgeons who are trained to use this systems. Furthermore, the surgery completion duration is reported to be increased. Another commercial system is named NeuroArm and it is the one of the most comprehensive passive robots until now, which is reported to be used in many surgeries [6]. It is able to perform stereotactic and microsurgery with real time MRI. Additionally, this system provides haptic feedback to the surgeon. However, among all the surgical systems, probably the most well-known one is the da Vinci ${ }^{\circledR}$ surgical system. Although its main use is in laparoscopic procedures, it is adapted for also transnasal endoscopic surgeries [7]. However, due to the large size of the system and complicated structure, it is not suitable for neurosurgical applications.

Pituitary tumor is a frequently observed tumor type, which causes important health problems from visual loss through hormonal imbalances [8]. In the management of this tumor, endoscopic endonasal pituitary surgery is a modern treatment method that is cost-efficient and more preferable than open-skull or microscopic transsphenoidal pituitary tumor surgeries [9]. In this surgery, to obtain 
internal images, an optical system called endoscope is inserted and directed by the surgeon starting from the nostril of the patient, through the naval cavity to the sphenoid sinus, which is right in front of the saddle-shaped hole named Sella Turcica in the skull base where the pituitary gland is located. The tumor is removed by the special surgical tools, which are also inserted through the nostrils [10]. Recent studies showed that endoscopic pituitary surgery leads to at least equal or even more tumor resection rates, better clinical results, higher patient comfort, less hospitalization duration and cost-efficiency in the long run compared to the microscopic surgery $[9,11,12]$. These mentioned reasons make endoscopic pituitary surgery increasingly more preferable among both surgeons and patients. One of the biggest problems in this operation is that the surgeon has to handle and control the endoscope during the whole surgery. This situation causes the surgeon to get tired and not to use the hand holding the endoscope to operate with other tools, which reduce the efficiency and prolongs the duration of the procedure that typically lasts for 2-4 hours. Some solutions have been offered to compensate for this problem such as having an assistant to handle the endoscope, using a fixed endoscope holder, endoscopes handled by robots that are controlled via variety of methods. In Figure 1, it can be observed that surgeon is using his left hand to hold the endoscope and the other hand to operate with a surgical tool while the assistant is helping him to use up to two more surgical tools. This type of procedure is needed very often during this type of surgery.

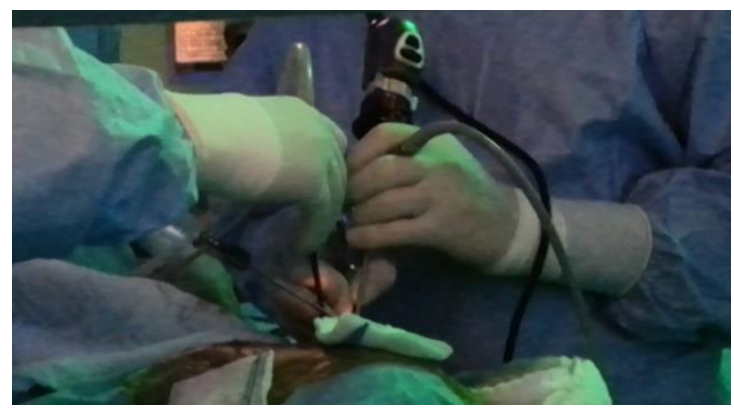

Fig. 1 Cooperation of the surgeon and the assistant throughout the surgery

Due to the limitations of the previously developed systems and including an assistant that is expected to follow the demands of the surgeon precisely, the use of these methods is problematic in clinical applications. The objective of our work is to provide a new robotic surgery system that enables the surgeon to use his/her two hands efficiently at the same time. This system is designated to be a masterslave teleoperation system to handle and control the positioning of the endoscope inside the surgery area. In this paper, among the other novel approaches for the proposed system, the determination of the information to be acquired and to be sent from the master system and the processing type of this information for the slave input is presented. Next section describes the previous work conducted on 
developing robotic systems to handle and direct optical systems in minimally invasive surgeries. The teleoperation architecture is explained in the third section which is followed by the preliminary experimentation result for the information exchange and control of the robot handling the endoscope. The paper is concluded with the discussions and conclusions based on the presented experimental results.

\section{Background on robotic endoscopic surgery}

A straightforward and quick solution for holding the endoscope during the surgery for 2-4 hours is to devise a stable holder which can be directed by the surgeon manually whenever required. Some of these endoscope holders are designed as passive holder mechanisms with simple mechanical joint locks such as Martin's Arm [13], ASSISTO [14], Endoboy [15], and TISKA [16]. Another type of holders has pneumatically controlled joint locks such as UNITRAC [17] and POINT SETTER [18], which also include mechanical balancing systems. Another passive mechanism presented in [19] is also mechanically balanced which can be positioned and oriented manually. All of these passive endoscope holders and other similar devices still need much effort from the surgeon or assistant whenever a change of view for endoscope is needed. Therefore, the endoscope holders are not the ultimate solution to maximize the efficiency of endoscopic surgeries.

In the survey of Nishikawa [20], positioning systems used in surgical robot systems are classified into three groups based on the level of autonomy: (i) nonautonomous systems, (ii) semi-autonomous systems, and (iii) full-autonomous systems. In another review presented in [21] an analysis on FDA approved robots devoted to endonasal surgery was conducted to expose the advantages and disadvantages of these systems. Taniguchi et al. [22] reported 27 different endoscopic surgical robots developed in between the years 1994-2009. Eight of them were used on humans, others were either experimented on animals or presented as a model. In most of these systems, a commercially available endoscope is directed by a robot arm which is controlled by the surgeon via a control-panel.

One of the oldest examples of the robot controlled imaging systems is developed by Taylor et al. [23]. This system is composed of a four-axis robotic arm and a joystick, which can easily plugged-in to laparoscopic instruments in order to control robot holding the camera. Even if joystick sounds a good and easily implementable solution, this system could not be commercialized widely because it degraded the performance of surgeon in using the instruments.

Another important example was developed by Casals et al. [24] for laparoscopic surgeries in which an industrial robot was used. In this system, force sensors are placed on the connections in order to prevent the dangerous situations for the patient. Surgeon controlled the camera by the input commands received from his/her head movements. However, it is difficult for the surgeon to keep his/her head stable throughout the surgery. Hence, this work also could not find use in surgeries. 
Narwell et al. [25] developed another method which uses a footswitch in order to control the robot handling the endoscope. However, this system also was not widely used since it did not provide a suitable way for the surgeon carry out the surgery without spending extra effort to control the robot. Wei et al. [26] offered another solution for all problematic points of previous works. In this system, a stereo camera acquires the motion of instruments used by surgeon and robot controlling the laparoscope mimics these motions. The surgery robot using this method, Aesop, is employed in over 1000 surgeries. However, this method relies on only stereo camera feedback and because of the resolution and speed limitations, it is not able to detect small and quick movements. Also, it reflects every motion of target instrument which may cause some dangerous situations during the surgery.

Researchers used various methods to acquire control demands from the surgeon to direct the endoscope during the surgery. There are some other examples in which the control demands are acquired by voice commands [27], by head motions [28], and by both joystick and voice commands [29]. In addition, KaLAR system [30] acquires the control demands by voice command and also includes an auto-tracking control. The commercially available VIKY system [31] has a foot pedal and voice recognition system to acquire control demands and it also uses image processing methods to make the robot follow the movements of surgical instruments. In Lapman robotic system [32], a joystick called LapStick ${ }^{\circledR}$ is fixed on a surgical tool as another way to acquire control demands. Emaro [33] is a commercially available air-powered surgical assistive robot that handles and controls the position of the endoscope by acquiring the control demands from the foot buttons and a gyroscope that is located on the forehead of the surgeon. The endoscope robot system FREE [34] is under development and in this system it is proposed to acquire the control demands from the gyroscope attached to the surgeon's foot. Infrared signals send from a headset worn by the surgeon is tracked and used as control demands in the EndoAssist system [35]. In 2010, Rilk et al. [36] fused all the control demand acquiring methods in the previous works (voice, footswitch etc.). In addition to this, in this system, endoscope images are processed and robot is driven to keep the surgical instruments in the middle of the display.

In the works discussed above, various ways are proposed and used to acquire control demands to drive the robot handling the endoscope. In the light of the previous experiences our aim is to develop a teleoperation system architecture in which the surgeon will be able to send control demands in an intuitive way with minimal effort of the surgeon. The next section describes the general teleoperation architecture to be used specifically for the endoscopic pituitary surgery.

\section{Teleoperation system architecture}

Generally, in teleoperation systems the master and slave robot systems are in different places but in this work, both robot systems are in the same place. In this tel- 
eoperation architecture, master system is a main control unit mounted on a surgical tool that the surgeon uses frequently during surgery, the aspirator. This main control unit is composed of two main parts as the 3-axis gyroscope and the electronic control board acquiring and transferring the sensor signals wirelessly to the slave system. Assuming that the surgeon uses his/her right hand for critical operations such as drilling, the main control unit is attached on and motions are proposed to be acquired from the aspirator tool used by the left hand of the surgeon.

The slave system is a robot arm whose end-effector is the endoscope. Robot arm is used in order to direct the endoscope only inside the surgical area. Different from the examples in literature, this slave robot arm is to be placed into the surgical area by the surgeon and retrieved back from the surgical area again by the surgeon whenever it is necessary. In this way, entering and retrieving processes, which are less critical in operation, can be done in a much faster way. Another advantage of this approach is that the system can be passively backdriven and retrieved from the surgical area in case of an unexpected failure.

The slave system consists of two main mechanisms; a weight-balanced six DoF carrier part, which can be passively backdriven by the surgeon and an active robot, which is driven by the control demands sent from the main control unit.

After the surgeon adjusts the endoscope image settings, the operating procedure of the system during the surgery is as follows:

1) The surgeon places the endoscope, which is attached to the robot, inside the surgical area. At this stage, brakes are on for all the actuators of the robot, which is positioned in the middle of its workspace. The demonstration of the surgeon placing the endoscope inside the surgical area is presented in Figure 2.

2) The main control unit is mounted on the aspirator to be used.

3) The surgeon activates the data flow between the main control unit and the slave system by pressing the activation button on main control unit whenever there is a need to change the direction of the endoscope. As long as the button pressed, main control unit remains active and it transfers the control demand information to slave robot. While the slave robot is driven to its required position, the compliant control algorithm implemented for the control of the robot prevents the transfer of unnecessary amounts of forces to the patient.

4) When the endoscope reaches the desired position, the activation button is released and the robot remains in that position.

5) When the lens of endoscope gets dirty, surgeon retrieves the endoscope from surgical area for cleansing. Then the 5-step procedure is repeated.

Taniguchi et al. [22] state that yaw, pitch and insertion (surge) motions are necessary motions of an endoscope inside the surgical area. It should be noted that in our system, the motions that require larger workspace and more DoF, such as entering the endoscope into the surgical area and retrieving it back from the surgical area, are accomplished by the surgeon manually by backdriving the 6 DoF carrier system. Therefore, the active slave robot system is designed to have 3 DoF motion identified as yaw, pitch and surge. In accordance, control demands from the surgeon should be acquired for these identified motions. 


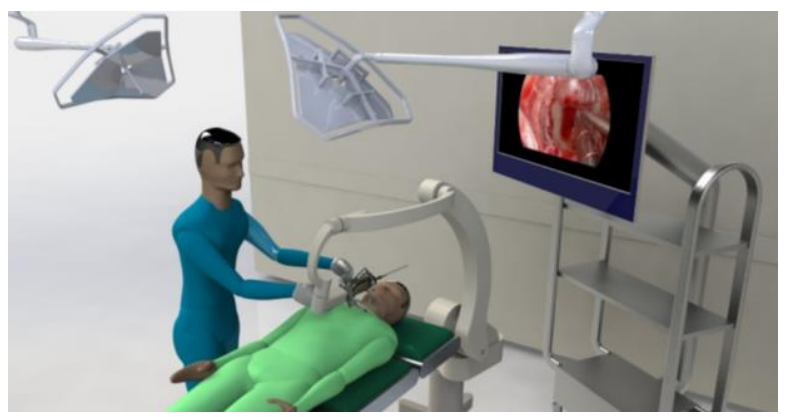

Fig. 2 Endoscope placement procedure

In order to issue control demands for the slave robot, surgeon activates the button placed on the main control unit and the orientation change is measured via the gyroscope in terms of angular velocity in three axes as pitch, roll and yaw. Angular velocities about yaw and pitch axes are used as control demand for slave robot about the same axes however; angular velocity measured about the roll axis is used for issuing control demand for the surge motion of the endoscope along its shaft direction. The representation of the information flow from the gyroscope axes to the 3 DoF robot arm is given in Figure 3. It should be noted that the presented mechanism of the slave robot is given for general representation of its motion capabilities. Nevertheless, the workspace of the slave is spherical and it is limited due to safety reasons after the analysis for the minimal required workspace.

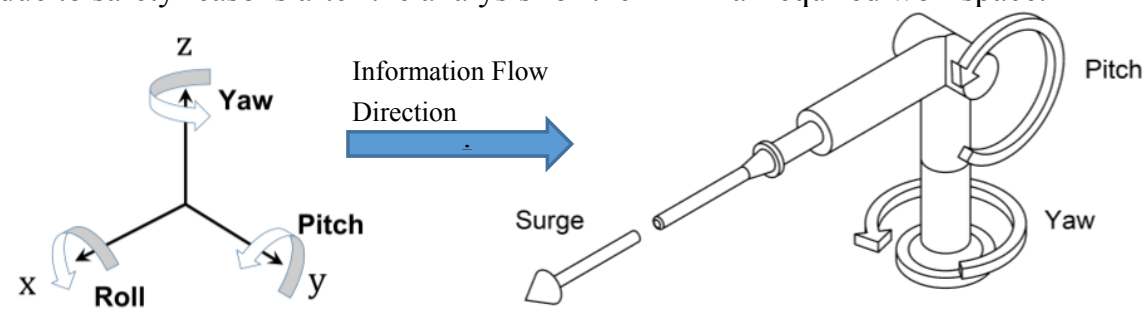

Fig. 3 The representation of information flow from the gyroscope axes to the 3 DoF slave robot arm

The next section describes the experimental setup to imitate the teleoperation architecture provided in this section to investigate the use of two different methods of controlling the endoscope robot.

\section{Experimentation Setup for Testing the Usability of the Teleoperation Architecture}

The experimental setup is composed of a replica of a surgical tool with an embedded gyroscope to be used as the master system and a virtual representation of the 
slave robot. The virtual slave robot is a spherical robot arm and a camera viewpoint is placed at the tip point of this robot arm. The surgeon handling the master system is shown in Figure 4(a). While the surgeon uses the replica of the surgical tool, he can also view the surgical area from the computer screen. This view is generated by the virtual camera placed at the tip of the slave robot. As the robot is moved by the processed control demands, the view of the virtual surgical area changes accordingly. This provides the sense that the surgeon is controlling a real robot that is handling the camera since during a surgery; the surgeon constantly looks at the endoscope image presented on a screen.

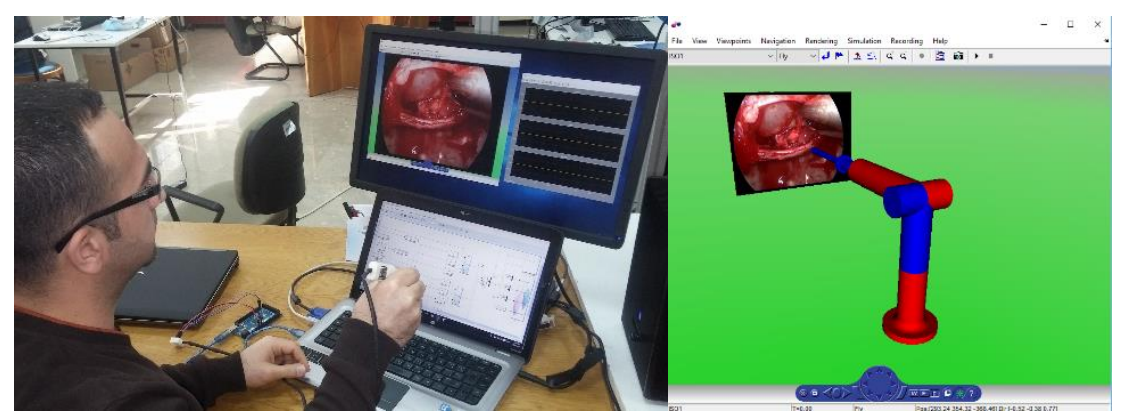

(a)

(b)

Fig. 4 (a) The surgeon handling master system while observing the endoscope's visual feedback (b) The virtual representation of the slave robot

During the experimentation, although the surgeon does not see the slave robot, it is shown in Figure 4(b) to provide a sense of how the virtual reality system works. The virtual slave robot presented in Figure 4(b) is the 3 DoF spherical robot arm and right in front of it, there is a screenshot of the surgical area. As there is a change in the orientation and the position of the robot's tip point, the portion of the visualized screenshot changes and this creates the effect of controlling the real endoscope robot.

The replica of the surgical tool, which is produced by using an additive manufacturing system, is shown in Figure 5. The main control unit on this tool is composed of a gyroscope unit and a push-button.

In the experimental setup, the gyroscope of the inertial measurement unit (IMU) named GY-88 MPU-6050 ${ }^{\mathrm{TM}}$ by MotionTracking ${ }^{\mathrm{TM}}$ Devices is used. This sensor offers four different sensitivity measures. In the scope of this work, resolution is relatively more important than full scale range; therefore, FS SEL $=0$ setting is selected to set the range as $\pm 250 \%$ sec and sensitivity as $131 \mathrm{LSB} /(\% / \mathrm{sec})$. In order to receive and process the measurements obtained from gyroscope, Arduino Mega 2560 board is used. I2C protocol is used for the communication between microprocessor and gyroscope in order to have a noise-free acquisition of the sensor measurements. The processed sensor measurements are forwarded to Matlab via serial port as control demands for the slave robot in real time by using Real 
Time Windows Target at a sampling frequency of $50 \mathrm{~Hz}$. This rate is enough for visualization of the surgical area. The connection details of the gyroscope and the push-button are shown in Figure 6. The LED is placed to warn the surgeon the push-button is pressed and the data is being transferred to the slave system.

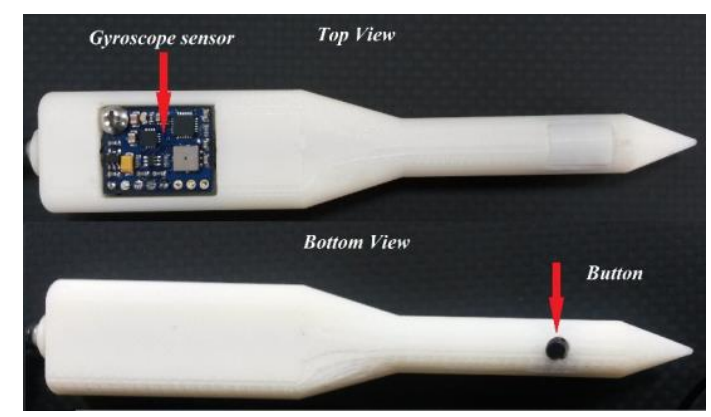

Fig. 5 Replica of a surgical tool and the main control unit's components

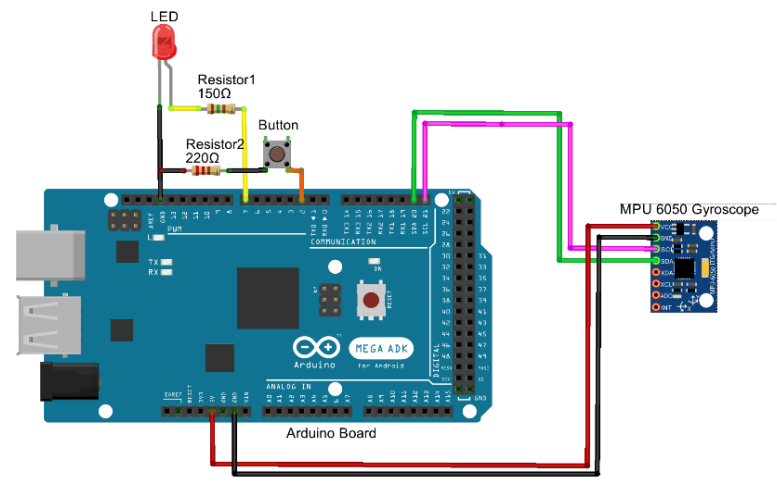

Fig. 6 Wiring of the gyroscope and the push-button to the Arduino board

\subsection{Main Control Unit's Algorithm}

The flowchart of the main control unit is shown in Figure 7. It is observed from the figure that the system initiates as soon as it is connected to the power. In the first step, I2C and serial communication protocols should be declared since all the wiring between the gyroscope and the Arduino board should be known by the microprocessor before receiving any signal. Then, the baudrate of serial communication is specified. After these, identification starts and gyroscope measurements are acquired and kept in predefined variables. This process repeats in each $80 \mathrm{~ms}$.

The push-button, which is presented in Figure 5 and 6, is used to either allow or prevent the data flow from the Arduino board to Matlab. The state of the button is checked continuously and if the button is pressed, data flow is allowed and the 
speed information is sent to Matlab. Otherwise, only "zero" value is sent through serial port. The reason of sending "zero" value instead of sending "nothing" is that the serial communication would be required to be reconfigured every time the push-button is pressed. Thus, it would degrade the performance of the system.

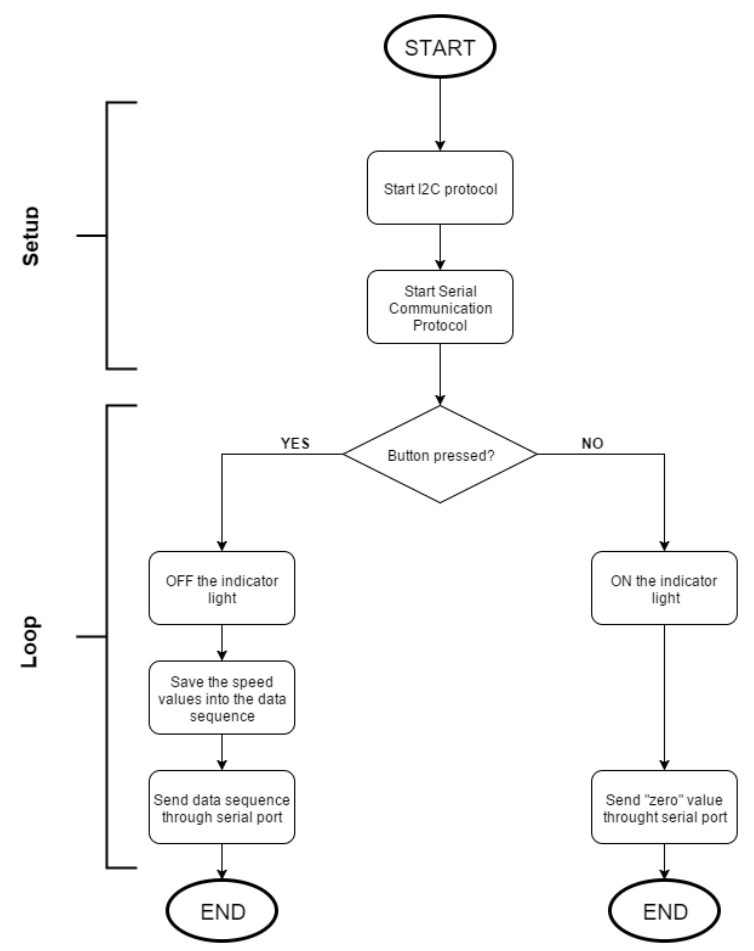

Fig. 7 The flowchart of the program on Arduino board

In the main control unit, there is also an indicator light (LED) to indicate the data flow condition. If the light is on, it means that the system is ready for the data to be sent but currently there is no data being sent. If the light is off, that means there is a loaded data flow over serial link. The situation of this light is dependent on the condition of the push-button. The program is terminated stopping the hardware-in-the-loop simulation built in Matlab Simulink.

\subsection{Teleoperation modes}

Although the acquired signal from the gyroscope is angular rate, while processing and converting this signal into control demands to drive the slave robot, two different methodologies can be applied. These methodologies are described as: 
Displacement-to-displacement method: In this method, the angular rate measured from the gyroscope is processed to find angular displacements in pitch, roll and yaw axes and they are mapped to the control demands for the slave robot in terms of angular displacements in pitch and yaw axes and translational displacement along the surge axis, respectively. Defintion of the axes is provided in Figure 3. The mathematical relationships to apply this method are given in (1), (2) and (3).

$$
\begin{gathered}
S_{i}=\operatorname{sat}\left(K_{S} \int R_{r} d t+S_{i-1}\right) ; i=0,1,2, \ldots \\
Y_{i}=\operatorname{sat}\left(K_{Y} \int Y_{r} d t+Y_{i-1}\right) ; i=0,1,2, \ldots \\
P_{i}=\operatorname{sat}\left(K_{P} \int P_{r} d t+P_{i-1}\right) ; i=0,1,2, \ldots
\end{gathered}
$$

As it is explained in the flowchart, each time the push-button is pressed, realtime gyroscope measurements are sent to the slave system and also pressing the push-button increases the $i$ count by 1 in (1), (2) and (3). In the initial conditions, $S_{0}, Y_{0}$ and $P_{0}$ values are set to zero and the $S_{l}, Y_{l}$ and $P_{l}$ values are updated as long as the push-button is pressed. After the push-button is released $S_{l}, Y_{l}$ and $P_{l}$ values are stored for the next time the push-button will be pressed. $S_{i}, Y_{i}$ and $P_{i}$ are the $i^{\text {th }}$ displacement demand along surge axis, $i^{\text {th }}$ angular displacement demands about the yaw and pitch axes, respectively. $R_{r}, Y_{r}$ and $P_{r}$ are the angular rates measured from the gyroscope about roll, pitch and yaw axes. Finally, $K_{s}, K_{y}$ and $K_{p}$ are the gains used scale the workspaces of the main control unit attached surgical tool and the slave robot handling the endoscope. If these gains are selected to be larger, then with a smaller motion of the main control unit attached surgical tool, a larger displacement demand for the slave robot will be issued. Additionally, saturation is used in (1), (2) and (3) in order to limit the workspace of the slave robot (to simulate the real case scenario).

Displacement-to-velocity method: In this method, the measured angular rates are processed to find angular displacements in pitch, roll and yaw axes and they are mapped to the control demands for the slave robot in terms of angular rates in pitch and yaw axes and translational velocity along the surge axis, respectively. The mathematical relationships to apply this method are given in (4), (5) and (6).

$$
\begin{aligned}
& S_{i}=\operatorname{sat}\left[\int\left(K_{S} \cdot \sigma \int R_{r} d t\right) d t+S_{i-1}\right] ; i=0,1,2, \ldots \\
& Y_{i}=\operatorname{sat}\left[\int\left(K_{Y} \cdot \sigma \int Y_{r} d t\right) d t+Y_{i-1}\right] ; i=0,1,2, \ldots \\
& P_{i}=\operatorname{sat}\left[\int\left(K_{P} \cdot \sigma \int P_{r} d t\right) d t+P_{i-1}\right] ; i=0,1,2, \ldots
\end{aligned}
$$


The parameters, initial conditions and the applications of the formulas for this method are almost the same with the previous method. The only difference is that in the first integrations, a reset signal is utilized to reset the result of the first integration in (4), (5) and (6) to zero whenever the push-button in the master side is released. Therefore, whenever the push-button is not pressed $\sigma$ values in (4), (5) and (6) becomes equal to 0 and otherwise it is equal to 1 . In this way it is guaranteed that the control demand in terms of velocity sent to the slave system is a zero command and the slave robot will not be operated until the push-button is pressed.

\section{Conclusions}

This work is dedicated to investigating the most suitable way of control demand acquisition from the surgeon in order to control an endoscope robot during the endoscopic pituitary surgery. Even before constructing the robot to control the endoscope, a simulation of the surgery is developed only to evaluate the ease of sending control demands to the slave robot, which is also called the endoscope robot in this work. Experimental setup is devised as a hardware-in the-loop simulation in which the control signals are acquired from a gyroscope and processed to drive a virtual slave robot with a camera that can replicate the visual information in the related surgery. In this study, there is no haptic feedback and the virtual robot is directed by only motion demands in a kinematics mode simulation environment. Therefore, $80 \mathrm{~ms}$ of sampling rate is sufficient to evaluate the teleoperation methods. Two different methods to drive the endoscope robot have been developed and the neurosurgeons from Hacettepe University tested the system with these methods. The first method is very similar to moving the cursor with the mouse on a screen. Although the workspace scaling ratios are increased, it was found to be requiring too much motion during a surgery. It should be recalled that the main controller with the gyroscope is mounted on the suction tube and this tool is inside the surgical area during the control of the endoscope robot. The displacement-to-velocity method is evaluated to be much easier to be operated with minimal effort especially when the workspace scaling ratio is increased. In this method, although the surgeon moves the suction tube in the required direction by a minimal amount and just stops there while pressing the push-button, the slave robot still continues to move the endoscope in the required direction. As a result of these evaluations, the displacement-to-velocity method was found to be the most convenient way of sending control demands to the endoscope robot.

\section{Acknowledgements}

This work is supported in part by The Scientific and Technological Research Council of Turkey via grant numbers 115E725 and 115E726. 


\section{References}

1. ROBODOC. “The ROBODOC ${ }^{\circledR}$ Surgical System” ROBODOC ${ }^{\circledR}$ :: Professionals, Available via ROBODOC. http://www.robodoc.com/professionals.html

2. Renishaw plc. "neuromate ${ }^{\circledR}$ ". neuromate ${ }^{\circledR}$ stereotactic robot, Available via RENISHAW. http://www.renishaw.com/en/neuromate-stereotactic-robot--10712

3. MAKO Surgical Corp. "MAKOplasty ${ }^{\circledR}$ ". MAKOplasty ${ }^{\circledR} \mid$ MAKO Surgical Corp. Available via Stryker. http://www.makosurgical.com/makoplasty

4. Sekhar, L. N., Tariq, F., Kim, L. J., Pridgeon, J. and Hannaford, B.: Commentary: virtual reality and robotics in neurosurgery. Neurosurgery, 72, A1-A6 (2013)

5. Mitsuishi, M., Morita, A., Sugita, N., Sora, S., Mochizuki, R., Tanimoto, K., Baek, Y.M., Takahashi, H. and Harada, K.: Master-slave robotic platform and its feasibility study for micro-neurosurgery. The International Journal of Medical Robotics and Computer Assisted Surgery, 9, 180-189 (2013)

6. Sutherland, G. R., Latour, I. and Greer, A. D.: Integrating an image-guided robot with intraoperative MRI. Engineering in Medicine and Biology Magazine, 27, 59-65 (2008)

7. Kupferman, M. E., DeMonte, F., Levine, N. and Hanna, E.: Feasibility of a robotic surgical approach to reconstruct the skull base. Skull Base-an Interdisciplinary Approach, 21, 79-82 (2011)

8. Levy A. Pituitary disease: presentation, diagnosis, and management. J Neurol Neurosurg Psychiatry. 75 Suppl 3:iii47-52 (2004)

9. Komotar, R. J., Endoscopic endonasal compared with microscopic transsphenoidal and open transcranial resection of giant pituitary adenomas. Pituitary, 15(2):150-159 (2012).

10.Laws ER, Wong JM, Smith TR, de Los Reyes K, Aglio LS, Thorne AJ, Cote DJ, Esposito F, Cappabianca P, Gawande A. A checklist for endonasal transsphenoidal anterior skull base surgery. J Neurosurg. 30:1-6 (2015).

11. Almeida, J. P., De Albuquerque, L. A., Dal Fabbro, M., Sampaio, M., Medina, R., Chacon, M., Gondim, J.: Endoscopic skull base surgery: evaluation of current clinical outcomes. Journal of neurosurgical sciences, (2015).

12. Berker, M., Işikay, I., Berker, D., Bayraktar, M., Gürlek, A.: Early promising results for the endoscopic surgical treatment of Cushing's disease. Neurosurgical review, 37(1), 105-114 (2014).

13. Marina Medical, Sunrise, FL. Available via marinamedical http://www.marinamedical.com/product/martins-arm-table-assistant-set

14. GEOMED Medizin-Technik, Tuttlingen, Germany. Available via geomed. http://www.geomed.de./index.php?id=60\&L $=1$

15. MIKROLAND Endobloc Company, Rue du Patis, France. Available via mikrolandendobloc. http://www.mikroland-endobloc.fr/gb/ index-gb.html

16. Schurr, M. O., Arezzo, A., Neisius, B., Rininsland, H., Hilzinger, H. U., Dorn, J., Roth, $\mathrm{K}$ and Buess, G. F.: Trocar and instrument positioning system TISKA. Surgical endoscopy, 13, 528-531 (1999)

17. Aesculap, Inc., Center Valley, PA. Available via Aesculap. https://www.aesculapusa.com/assets/base/doc/DOC905_Unitrac_Retraction_and_Holdi ng_Brochure.pdf

18. MITAKA KOHKİ CO., LTD., Japan. Available via karlstorz. https://www.karlstorz.com/cps/rde/xbcr/karlstorz_assets/ASSETS/2149000.pdf

19. Kuo, C. H. and Lai, S. J.: Design of a Novel Statically Balanced Mechanism for Laparoscope Holders With Decoupled Positioning and Orientating Manipulation. Journal of Mechanisms and Robotics, 8, 015001-015001-10 (2016) 
20. Nishikawa, A.: The Surgeon-Robot Interface for Controlling the Position of a Laparoscope. Japan Society of Computer Aided Surgery, 6, 69-74 (2004)

21. Trévillot, V., Garrel, R., Dombre, E., Poignet, P., Sobral, R. and Crampette, L.: Robotic endoscopic sinus and skull base surgery: review of the literature and future prospects. European annals of otorhinolaryngology, head and neck diseases, 130, 201-207 (2013)

22. Taniguchi, K., Nishikawa, A., Sekimoto, M., Kobayashi, T., Kazuhara, K., Ichihara, T., Kurashita, N., Takiguchi, S., Doki, Y., Mori, M. and Miyazaki, F.: Classification, design and evaluation of endoscope robots. Robot Surgery. Editor: Baik, S. H. Rijeka: InTech (2010)

23. Taylor, R.H., Funda, J., Eldridge, B., Gomory, S., Gruben, K., LaRose, D., Talamini, M., Kavoussi, L. and Anderson, J.: A telerobotic assistant for laparoscopic surgery. Engineering in Medicine and Biology Magazine, IEEE, 14, 279-288 (1995)

24. Casals, A., Amat J. and Laporte E.: Automatic Guidance of an Assistant Robot in Laparoscopic Surgery. In: Proceedings of the IEEE Conference on International Conference on Robotics and Automation, Minneapolis, Minnesota, 1, pp. 895-900 (1996)

25. Narwell, N. I., Weker, D. R., Wang Y. 1994. "A Force controllable macro-micro manipulator and its application to medical robotics". JPL Computer Motion Inc. Rapor no: N94- 30441.

26. Wei, G. Q., Arbter, K. and Hirzinger, G.: Real-time visual servoing for laparoscopic surgery. Controlling robot motion with color image segmentation. Engineering in Medicine and Biology Magazine, IEEE, 16, 40-45 (1997)

27. Wang, Y., Laby, K. P., Uecker, D. R., Mangaser, A. A. and Ghodoussi, M.: Automated Endoscope System For Optimal Positioning. Patent no: US5878193 (1999)

28. Nishikawa, A., Hosoi, T., Koara, K., Daiji, N., Hikita, A., Asano, S., Miyazaki, F., Sekimoto, M., Miyake, Y., Yasui, M. and Monden, M.: Real-time visual tracking of the surgeon's face for laparoscopic surgery. Medical Image Computing and ComputerAssisted Intervention - MICCAI 2001. Editors: Niessen, W. J., Viergever and M. A., pp. 9-16 (2001)

29. Buess, G. F., A. Arezzo, M. O. Schurr, F. Ulmer, H. Fisher, L. Gumb, T. Testa and C. Nobman.: A new remote-controlled endoscope positioning system for endoscopic solo surgery. Surgical endoscopy, 14, 395-399 (2000)

30. Lee, Y. J., Kim, J., Ko, S. Y., Lee, W. J. and Kwon, D. S.: Design of a compact laparoscopic assistant robot: KaLAR. In Proceedings of the International Conference on Control Automation and Systems, pp. 2648-2653 (2003)

31. VIKY, EndoControl, Inc., Dover, DE. Available via endocontrol-medical. http://www.endocontrol-medical.com

32. Lapman, Available via medsys. www.medsys.be/documents/lapman_leaflet.pdf

33. Emaro, The World's First Pneumatic Endoscope Manipulator Holds Promise for Quality Surgery. Available via Tokyo Institute of Technology. http://www.titech.ac.jp/english/news/pdf/tokyotechpr20150731_emaro_en.pdf

34. Chan, J.Y., Leung, I., Navarro-Alarcon, D., Lin, W., Li, P., Lee, D.L., Liu, Y.H. and Tong, M.C.: Foot-controlled robotic-enabled endoscope holder (FREE) for endoscopic sinus surgery: A cadaveric feasibility study. The Laryngoscope, 126, 566-569 (2016)

35. Aiono, S., Gilbert, J. M., Soin, B., Finlay, P. A. and Gordan, A.: Controlled trial of the introduction of a robotic camera assistant (Endo Assist) for laparoscopic cholecystectomy. Surgical Endoscopy and Other Interventional Techniques, 16, 1267-1270 (2002)

36. Rilk, M., Kubus, D., Wahl, F. M., Eichhorn, K. W., Wagner, I. and Bootz, F.: Demonstration of a prototype for robot assisted Endoscopic Sinus Surgery. In Robotics and $\mathrm{Au}-$ tomation (ICRA), 2010 IEEE International Conference on, Anchorage, AK, pp. 10901091 (2010, May). 